\title{
Evaluation of antibiotic treatment and antibiotic de-escalation in a intensive care unit with selective digestive decontamination
}

\author{
C Sánchez Ramirez*, L Caipe Balcázar', S Hípola Escalada', MA Hernández Viera'', M Cabrera Santana', \\ N Sangil Monroy², A Bordes Benitez ${ }^{3}$, P Saavedra Santana ${ }^{4}$, S Ruiz Santana ${ }^{1}$
}

From ESICM LIVES 2015

Berlin, Germany. 3-7 October 2015

\section{Objective}

To evaluate the appropriate use of antibiotics and their de-escalation (DE) to treat nosocomial infections in an Intensive Care Unit (ICU) with Selective Digestive Decontamination (SDD).

\section{Method}

In a polyvalent ICU of 30 beds from October 1, 2011 to September 30, 2014 nosocomial infections (pneumonia, urinary tract infections, catheter-related bacteremia (BRC) and of unknown origin and secondary nosocomial bacteremia) were prospectively collected. ENVIN-HELICS diagnostic criteria were applied. Etiology, inflammatory response to infection, antibiotic treatment (ATB T), and treatment modifications according to culture results, was analyzed. SDD was applied to all admitted patients requiring endotracheal intubation over 48 hours. For each of the groups categorical variables were summarized as frequencies and percentages and number in means and standard deviations (SD) or median with interquartile ranges (IQR). Percentages were compared, as appropriate, with the Fisher's exact test.or $\mathrm{X}^{2}$ test and medians with the Wilcoxon test for independent samples. For those variables that were associated with $\mathrm{DE}$ in the univariate analysis were entered into a logistic multidimensional analysis. The model obtained was expressed by $\mathrm{p}$-values and odd-ratios, which were estimated by confidence intervals at $95 \%$. A hypothesis test was considered statistically significant when p-value was less than .05 .

University Hospital of Gran Canaria Dr Negrín, Intensive Care Unit, Las

Palmas de Gran Canaria, Spain

Full list of author information is available at the end of the article

\section{Results}

Fifty-seven patients had ATB DE and 126 did not. There were no significant differences in demographics or type of admission (Figure 1)

Mortality was lower in patients receiving DE antibiotic (АТB) $(22.9 \%$, p: 0.095). In the multivariate study of urinary tract infection, septic shock or severe sepsis and secondary bacteremia were significant (Figure 2).

Of the 253 nosocomial infections ATB DE were performed at least in one of ATB in 131 (51.8\%) of them. The inflammatory response and the type of infection did not show any decrease in the group with DE compared to all infections. The ATB T was inadequate in 43 infections (16.9\%) (15 pneumonias, 11 urinary tract infections, 14 CRB and 3 secondary bacteremias). Targeted therapy was performed at least 1 time out of 109 infections (43.1\% of infections), 28 pneumonias, 35 urinary tract infections, 38 CRB and 10 BRC secondary bacteremias. The number of antibiotics used was 483 and in 156 occasions ATB DE was performed. Frequency of use and DE is shown in Figure 3.

\section{Conclusions}

Patients with received versus those that did not received DE had a tendency towards a significant lower mortality. The factors independently associated to DE were urinary and secondary bacteremia and septic shock or severe sepsis. Inadequate ATB $\mathrm{T}$ in our ICU happened in $16.9 \%$ of nosocomial infections. ATB DE was performed in $51.8 \%$ of them. The most commonly used antibiotics were piperacillin-tazobactam (29.6\%), levofloxacin (12.2\%) and meropenem (10.7\%). 


\begin{tabular}{|c|c|c|c|c|}
\hline & & Denesc & & $p$ \\
\hline & & $\mathrm{Si}$ & No & \\
\hline & & $N=57$ & $N=126$ & \\
\hline & Age, years & $58.3 \pm 17.2$ & $61.2 \pm 16.2$ & 260 \\
\hline & Male / Femde, \% & $68.4 / 31.6$ & $61.1 / 389$ & 342 \\
\hline & Apache-II & $19(15 ; 26)$ & $22(17 ; 29)$ & .062 \\
\hline & Glasgow Coma Score & $14(8 ; 15)$ & $15(9 ; 15)$ & 653 \\
\hline & Traumábic patients, $n(\%)$ & $9(15.8)$ & $15(11.9)$ & .471 \\
\hline & ICU stay, days & $34.5(18.5 ; 41.5)$ & $30(16.5 ; 48)$ & .806 \\
\hline & Deatsis , $n\{(\%)$ & $8(22.9)$ & $30(39.0)$ & .095 \\
\hline & Diabetes mellitus, $\mathrm{n}(\%)$ & $20(35.1)$ & $37(29.4)$ & .439 \\
\hline & Coronary patients, $n(\%)$ & $11(19.3)$ & $19(15.1)$ & .475 \\
\hline & Liver cirhosis, $n(\%)$ & $2(3.5)$ & $11(8.7)$ & .351 \\
\hline & Renal failure, $n(\%)$ & $15(26.3)$ & $32(25.4)$ & .895 \\
\hline & $\mathrm{COPD}, \mathrm{n}(\%)$ & $8(14,0)$ & $18(14,3)$ & $\$ 64$ \\
\hline & Emergency surgery, $n(\%)$ & $24(42.1)$ & $33(26.2)$ & .031 \\
\hline & Immunosuppression, $n(\%)$ & $5(8.8)$ & $11(8.7)$ & 993 \\
\hline & Neutropenia, $\mathrm{n}(\%)$ & $1(1.8)$ & $4(32)$ & 1 \\
\hline & Parenteral nutrition, $n(\%)$ & $13(22.8)$ & $29(23.0)$ & 975 \\
\hline & Renal mplacement therapy. $\mathrm{n}(\%)$ & $18(31.6)$ & $46(36.5)$ & .517 \\
\hline & Nosocomial pneumonia, $n(\%)$ & $29(50.9)$ & $46(36.5)$ & .067 \\
\hline & Cathater related Bacteremias, $n(\%)$ & $28(49-1)$ & $41(32.5)$ & .032 \\
\hline & Sacondary bacteremias, $\mathrm{n}(\%)$ & $4(7.0)$ & $30(23.8)$ & .007 \\
\hline & Urinary infections, $n(\%)$ & $10(17.5)$ & $43(34.1)$ & .022 \\
\hline & Inflammatory response & & & 015 \\
\hline & No & $1(1.8)$ & $9(7.1)$ & \\
\hline & Sepsis & $11(19.3)$ & $44(34.9)$ & \\
\hline & Severe sepsis & $4(7.0)$ & $14(11.1)$ & \\
\hline & Septic Shock & $41(719)$ & $59(46.8)$ & \\
\hline & $\mathrm{N}$, number; COPD.,Chronic Obstructive Pulmans & Disease & & \\
\hline
\end{tabular}

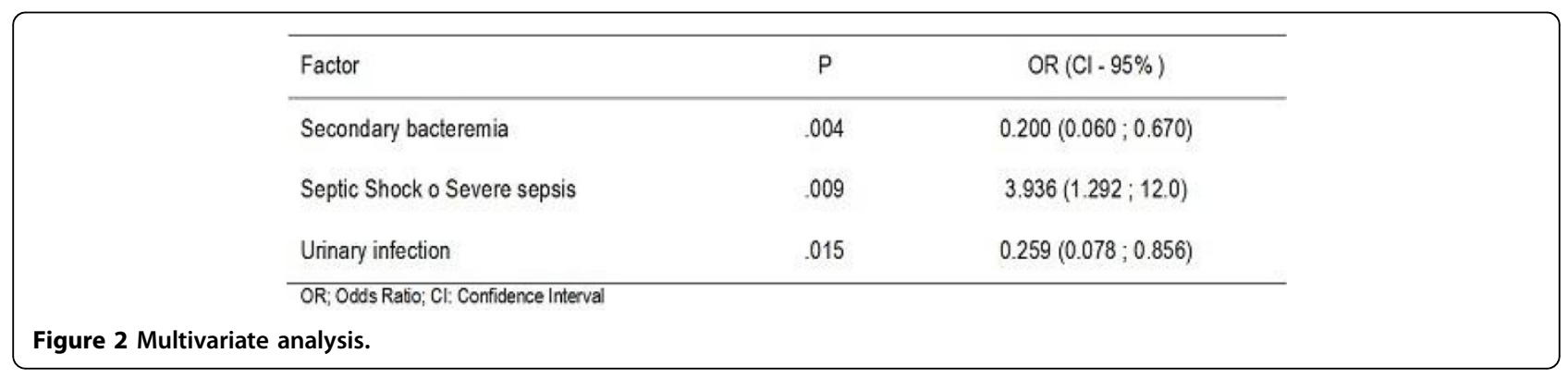




\begin{tabular}{|c|c|c|}
\hline \multicolumn{3}{|c|}{ Frequency } \\
\hline Antibiotic & of uso & Frecuency of de-escalation \\
\hline Amikacin & 11 & 3 \\
\hline Ampicilin & 2 & 0 \\
\hline Amoxicilin-clavulanabe & 1 & 1 \\
\hline Cefotaxime & 11 & 3 \\
\hline Ceftazidime & 21 & 6 \\
\hline Coftriaxon & 2 & 2 \\
\hline Cefuroxime & 1 & 1 \\
\hline Ciprofoxacin & 16 & 10 \\
\hline Cloxacilina & 10 & 2 \\
\hline Colistin & 18 & 2 \\
\hline Cotrinoxazole & 4 & 0 \\
\hline Dorycycline & 1 & 0 \\
\hline Erythromycin & 1 & 0 \\
\hline Flusonazole & 20 & 9 \\
\hline Fosfomicina & 10 & 5 \\
\hline Gontamicin & 9 & 4 \\
\hline Imipenem & 17 & 7 \\
\hline Metronidazole & 1 & 0 \\
\hline Penicillin & 1 & 1 \\
\hline Piperaclin-Tazobactam & 64 & 19 \\
\hline Ampicilin-Sulbactam & 1 & 1 \\
\hline Teicoplanin & 14 & 4 \\
\hline Tobramicyn & 10 & 2 \\
\hline Vancomycin & 15 & 9 \\
\hline Cefepime & 47 & 17 \\
\hline Meropenem & 52 & 19 \\
\hline Levolloxacin & 59 & 16 \\
\hline Grepafloxacin & 1 & 1 \\
\hline Linezolid & 47 & 9 \\
\hline Voniconazde & 2 & 2 \\
\hline Caspofungin & 8 & 0 \\
\hline Tigecycline & 2 & 1 \\
\hline Total & 483 & 156 \\
\hline
\end{tabular}

Figure 3 Antibiotics.

\section{Authors' details}

${ }^{1}$ University Hospital of Gran Canaria Dr Negrín, Intensive Care Unit, Las Palmas de Gran Canaria, Spain. ${ }^{2}$ University Hospital of Gran Canaria Dr Negrín, Pharmacy Department, Las Palmas de Gran Canaria, Spain.

${ }^{3}$ University Hospital of Gran Canaria Dr Negrín, Microbiology Department, Las Palmas de Gran Canaria, Spain. ${ }^{4}$ University of Las Palmas de Gran Canaria, Mathematics and Informatics Deparment, Las Palmas de Gran Canaria, Spain.
Published: 1 October 2015

doi:10.1186/2197-425X-3-S1-A2

Cite this article as: Sánchez Ramirez et al:: Evaluation of antibiotic treatment and antibiotic de-escalation in a intensive care unit with selective digestive decontamination. Intensive Care Medicine Experimental 2015 3(Suppl 1):A2. 